\title{
Effects of Farnesol on Drug-Resistant and Non-Resistant Candida albicans: Implications for Cosmetic and Pharmaceutical Applications
}

\author{
Neela Yar', Emma Wittman'1, Dominic Schaut ${ }^{1}$, Francesco De Seta ${ }^{2}$, Bryan Larsen ${ }^{1 *}$ \\ ${ }^{1}$ College of Osteopathic Medicine, Marian University Indianapolis, Indianapolis, IN, USA \\ ${ }^{2}$ Department of Medical Sciences, University of Trieste, Institute for Maternal and Child Health-IRCCS, BurloGarofolo, Trieste, \\ Italy \\ Email: *blarsenphd@msn.com
}

How to cite this paper: Yar, N., Wittman, E., Schaut, D., De Seta, F. and Larsen, B. (2020) Effects of Farnesol on Drug-Resistant and Non-Resistant Candida albicans. Implications for Cosmetic and Pharmaceutical Applications. Advances in Microbiology, 10, 383-396.

https://doi.org/10.4236/aim.2020.108028

Received: July 25, 2020

Accepted: August 18, 2020

Published: August 21, 2020

Copyright $\odot 2020$ by author(s) and Scientific Research Publishing Inc. This work is licensed under the Creative Commons Attribution International License (CC BY 4.0).

http://creativecommons.org/licenses/by/4.0/

\begin{abstract}
Background: Farnesol is added to numerous consumer products that intentionally, or inadvertently come in contact with tissues that may harbor the opportunistic yeast, Candida albicans. Objective: This study explores biological consequences of the exposure of Candida albicans from community infections or from a panel of antifungal drug resistant organisms on growth and survival of these organisms when exposed to farnesol. Methods: ATCC supplied Candida albicans from the MP8 drug resistance panel and an additional 12 strains of community-acquired Candida albicans were cultured in the presence of farnesol. With standard micobiologic techniques and flow cytometry evaluation, a series of experiments considered growth, morphology, viability and entrance into the quiescent persister phenotype of Candida with emphasis on differences between drug resistant and community organisms. Results: Differences growth yield, relative cell size and heat susceptibility distinguished the community organisms from the drug-resistant organisms. Using a subset of these organisms, exposure to farnesol resulted in diminished growth, inhibited hyphal growth, diminished cell membrane integrity and increased heat stress susceptibility. Data provided suggest that exposure to farnesol pushes cultures of Candida albicans toward the quiescent persister phenotype. Conclusion: Exposure of drug resistant and community strains of Candida albicans are modestly affected by farnesol in ways that may lessen their pathogenic potential. In contrast, the tendency of farnesol to engender greater numbers of quiescent organisms could support persistence of Candida.
\end{abstract}

\section{Keywords}

Farnesol, Candida albicans, Antifungal Resistance, Persister Phenotype, Quorum Sensing 


\section{Introduction}

Following the discovery of the role farnesol plays as a quorum-sensing autoinducer for the opportunistic yeast, Candida albicans, numerous studies on the molecular details of its role in survival, virulence and morphogenesis have been published [1]. In addition, other findings relevant to farnesol include modulation of host immune responses and effects on bacteria which may both enhance or diminish the course of infection [2].

Against this backdrop of many biological activities that have been attributed to farnesol, its industrial use as a perfume or component of cosmetics and household products and potentially in pharmaceutical preparations has occurred with limited apparent appreciation for biological side effects (beneficial or not) that might result from its addition to formulations of commercial products. Farnesol is also a component of essential oils which are also used commercially and hence due to public interest and acceptance, have widening commercial use. Consumers of these products may be attracted to them out of desire for products considered to be natural or which contain botanical substances in the formulation. Little direct tissue toxicity has been described for farnesol, and it is categorized by the US FDA as "GRAS" or generally recognized as safe. Reports of allergy to farnesol are rare [3] with only 22 of 2021 individual patch-tested showing a reaction. Incorporation of farnesol into a mucoadhesive vaginal gel has been reported [4].

While many of the biologically relevant effects such as quorum sensing occur at micromolar concentrations [5] [6] [7] [8] [9], consumer products may contain farnesol at higher levels and we report here findings obtained with Candida $s p$. to illuminate what benefits or concerns should be considered by the industrial use of farnesol. Specifically, this report explores potential differences between Candida strains that were known to have specific resistance patterns compared to community-derived organisms in terms of growth and fitness under stress.

\section{Methods}

\subsection{Microorganisms}

Fungal cultures available for this study included 14 strains comprising the ATCC MP8 drug-resistant panel which included 12 multi-resistant strains and two non-resistant (control) strains of Candida albicans. This panel was selected as the organisms are commercially available and contain well characterized resistance characteristics that allow for repeatability among laboratories and involve resistance to multiple drug classes. In addition, 12 Candida albicans strains were provided by a local clinical laboratory representing isolates from various body sites. Except where specifically designated, for logistical reasons, a subset of the above organisms was used.

The subset of organisms included three antifungal drug-resistant organisms from the ATCC MP-8 panel. These resistant strains were: ATCC 64124 Darlington (here labeled CA-A), ATCC 102313247 (here labeled CA-B) and ATCC 
28121304 (here labeled CA-C). Three community isolates had previously been identified by local clinical laboratories as Candida albicans using standard diagnostic microbiology methods and lacked known antifungal resistance. These were labeled as CA-1, CA-2 and CA-3.

All Candida strains were aliquoted and stored in 50\% glycerol and kept at $-80^{\circ} \mathrm{C}$ until needed and were grown overnight as starter cultures in brain heart infusion (BHI) broth for each experiment. Starter cultures were incubated overnight at $37^{\circ} \mathrm{C}$ in $5 \% \mathrm{CO}_{2}$.

\subsection{Experimental Design}

Experimental conditions were inoculated with approximately $1 \times 10^{4}$ organisms and were incubated overnight at $37^{\circ} \mathrm{C}$ in $5 \% \mathrm{CO}_{2}$ before experiment readout.

Experiments containing farnesol (Alfa Aesar-Thermo Fischer, Ward Hill MA, mixed isomers 96\%) were prepared by diluting stock from the manufacturer in absolute ethanol for addition to brain heart infusion broth. For plating medium, farnesol was added directly while the agar was molten, mixed quickly and poured into plates. In addition to BHI agar, we also employed modified spider medium (Brain Heart infusion agar was purchased from Difco BD and contained brain-heart infusion powder, sodium chloride, disodium phosphate with 1.5\% agar and dissolved according to manufacturer instructions, to which $1 \mathrm{~g}$ of mannitol/100ml of media was added and then autoclaved before dispensing into plates) for preliminary observations.

Absolute counts of yeast employed flow cytometry performed using the Becton-Dickenson Accuri C6 instrument. When flow cytometer counts were unavailable, turbidity, measured spectrophometrically at $450 \mathrm{~nm}$, was employed. Experiments were conducted in styrene well plates and unless otherwise noted, contained BHI as the background medium with or without farnesol. For analysis of the organisms after overnight incubation, each well was vigorously mixed before removing a $100 \mathrm{uL}$ aliquot which was added to $900 \mathrm{ul}$ of water containing propidium iodide $(60 \mathrm{mg} / \mathrm{ml}$ stock solution diluted $100 \mathrm{uL} / 50 \mathrm{~mL}$ sterile water) and analyzed for events in the forward scatter channel (FSC) of the cytometer. The C6 instrument allows absolute quantitation due to a fluidics system that accurately pumps the sample onto the flow cell rather than using air pressure to introduce the sample into the flowing sheath fluid.

Microbial counts were based on events triggered in the FSCchannel and relative particle size was indicated by mean FSC-h for each sample. Propidium iodide staining was observed in the orange fluorescence (FL-3h) channel and provided a measure of relative cell integrity.

\subsection{Heat Challenge}

Heat stress testing was done by placing $100 \mathrm{uL}$ aliquots from cultures conditioned in media with or without farnesol, placed in a heat block at $50^{\circ} \mathrm{C}$ for 20 minutes and thereafter immediately diluted in water containing propidium iodide. For most samples fungal enumeration was based on at least 2000 flow cytometer 
counts. Baseline propidium iodide staining was performed on cultures before heat treatment and the percent of cells with FL-3 signal above $1 \times 10^{3}$ was recorded. As second sample was similarly evaluated immediately after heat exposure and a heat stress index was calculated by $\%$ of cells staining with propidium iodide after heating divided by the percent of cells staining with propidium iodide before heating which provided the fold increase in staining resulting from heat stress.

\subsection{Persister Phenotype Estimation}

Density fractionation of yeast cultures was performed according to the method of Quasem, et al. [10]. Briefly, indixanol 60\% (Sigma Chemical, St. Louis MO) was diluted with an equal volume of tris buffered saline. Diluted indixanol (0.5 $\mathrm{ml}$ ) was pipetted into a microcentrifuge tube and the yeast culture layered atop the density cushion. Separation of high and low buoyancy cells was obtained by centrifuging at $2500 \mathrm{xg}$ for 2.5 minutes, then rotating the tube in the angle rotor and centrifuging an additional 2.5 minutes. The pellet is expected to be enriched with quiescent cells while the upper band is enriched for actively growing yeast as shown in Figure 1.

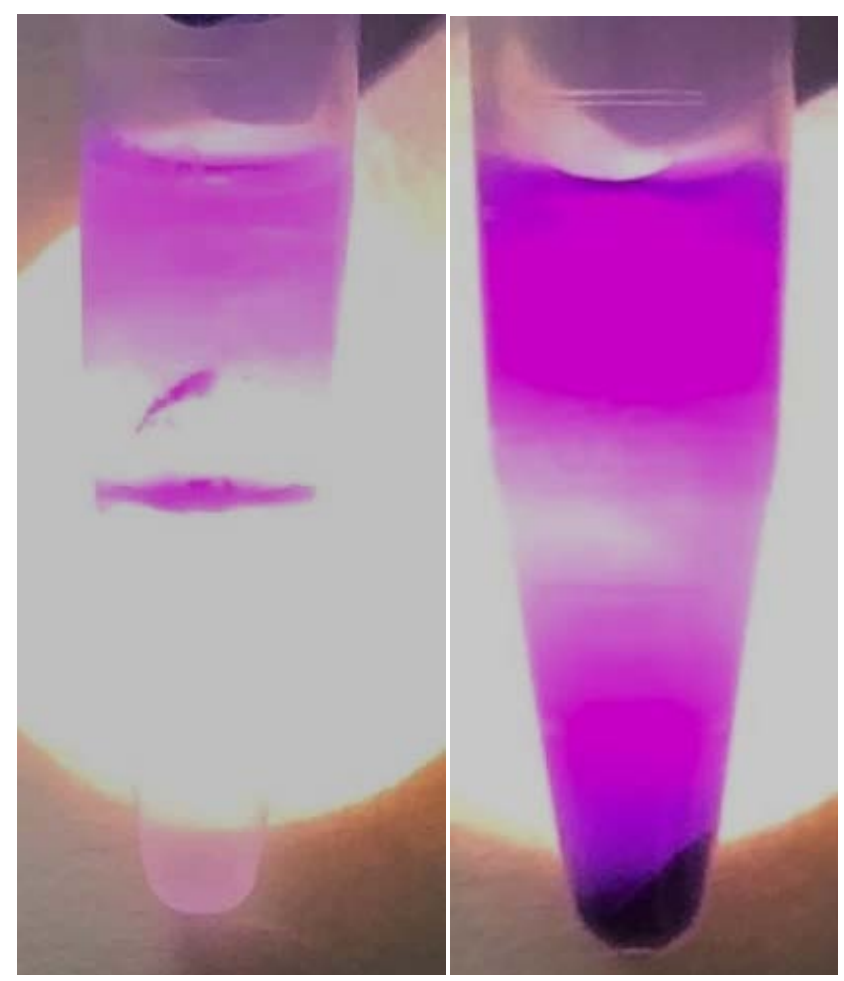

Figure 1. Yeast cultures were stained with crystal violet and washed prior to resuspension and density separation on indixanol density cushion. After separation the tubes were backlit to enhance visualization of upper and lower fractions. At left is a tube dominated by non-quiescent yeast and the image right shows a culture with a preponderance of quiescent and dead cells. Staining of the culture before separation was not used for the experiments, the staining was done here for illustrative purpose. With sufficiently high concentration of cells, the unstained bands could be visualized without staining. 


\subsection{Statistical Analysis}

Where appropriate, continuous variables were compared by two sample $\mathrm{T}$ test was applied and where possible paired comparisons were made. The level of significance was 0.05 .

Evaluation of generation time was calculated using the free online program at http://endmemo.com/.

\section{Results}

\subsection{Colony Morphology}

As an initial overview of the behavior or Candida albicans in the presence of farnesol, the entirety of the two sets of test organisms (drug-resistant and community acquired) were examined. BHI agar with or without farnesol and modified spider medium were prepared and $10 \mathrm{uL}$ aliquots of overnight cultures of each of the available test organisms were spotted onto these media with and without the addition of $0.3 \%$ farnesol. Plates were incubated at room temperature and monitored daily for 5 days for growth and the spider phenotype (mycelial growth) under each condition. Regardless of drug resistance, all strains tested grew on all media. However, the colony size on the farnesol-containing plates showed growth restriction compared to the non-farnesol plates and the appearance of spidering colonies was not present on farnesol-containing plates. No gross differences in the colony appearance of drug resistant and non-resistant strains were noted in this experiment.

While it was possible to evaluate the entire set of Candida isolates on a single plate by this method, the attempt to incorporate the hydrophobic farnesol into agar medium resulted in development of droplets of farnesol atop the medium, limiting its utility for subsequent studies.

\subsection{Strain Characterization}

Despite the lack of obvious differences between the drug-resistant and community strains in the initial study, a more detailed examination of intrinsic fitness of each group prior to further detailed studies of farnesol was undertaken. Accordingly, several aspects of growth were evaluated by flow cytometry. The 24-hour growth yield, relative organism size (based on mean FSC statistics), generation times over a 4-hour period and baseline propidium iodide staining and increase in propidium iodide staining after a 20-minute heat challenge are shown in Table 1.

The above experiment indicated that some, but not all of the parameters measured, differed significantly between the two groups of organisms, but the apparently smaller volume and number of cells after 24 hours in culture and the greater apparent membrane fragility before and after heating suggested that the experiments that followed should consider examples from both the drug-resistant and the community organisms available. The first three Candida albicans strains from each set (CA-A, CA-B and CA-C from the resistant panel and CA-1, CA-2 and CA-3 from the community strain set) were selected for further study. 
Table 1. Data summary for comparison of MP8 resistant organisms with community organisms including MP8 controls.

\begin{tabular}{cccccc}
\hline \multirow{2}{*}{ Property } & \multicolumn{2}{c}{ Resistant Isolates } & \multicolumn{2}{c}{ Community Isolates } & \multirow{2}{*}{$\mathrm{P}=$} \\
\cline { 2 - 5 } & Mean & SD & Mean & SD & \\
\hline $\begin{array}{c}\text { Growth Yield } 24 \mathrm{~h} \\
\text { Relative Size }\end{array}$ & $979 / \mathrm{uL}$ & 430 & 2335 & 2131 & 0.053 \\
$\begin{array}{c}\text { (as mean FSC-h value) } \\
\text { Biomass Index units }\end{array}$ & $1.51 \times 10^{6}$ & $0.143 \times 10^{6}$ & $1.22 \times 10^{6}$ & $0.27 \times 10^{6}$ & 0.008 \\
$\begin{array}{c}\left.\text { (Events } \times \text { mean FSC-h/1 } \times 10^{-6}\right) \\
\text { Generation Time }\end{array}$ & 1487 units & 654 units & 2533 units & 1714 units & 0.104 \\
Percent PI Staining & 55.52 min & ND & 56.43 & ND & 0.880 \\
Heat Stress Index & $17.3 \%$ & $6.18 \%$ & $11.65 \%$ & $4.92 \%$ & 0.035 \\
\hline
\end{tabular}

Growth yield is events/uL (FSC of BHI culture diluted 1:10). Relative size is average FSC height signal for each culture. Biomass index is the product of events/uL and mean forward scatter (without exponent). Generation time was determined after 4 hours of freshly inoculated culture and was based on forward scatter events evaluated using EndMemo (endmemo.com). PI staining was determined as the percent of events having FL3 channel signal above $1 \times 10^{3}$ based on data from unstained yeast. Heat stress index represents the fold increase in \%PI staining after exposure to $50^{\circ} \mathrm{C}$ for 20 minutes. Reported values represent the average of the individual Candida strains tested.

Comparisons for 24-hour growth yield and PI staining were measured for each strain and results are shown in Figure 2.

These data indicate variations among strains and when comparing 24-hour mean counts between resistant strains CA-A, CA-B, CA-C, to community strains CA-1, CA-2, CA-3, the community strains furnished slightly more robust growth $(\mathrm{p}=0.03$, students $\mathrm{T}$ test). The intrinsic PI staining trended higher for the resistant organisms but did not reach statistical significance $(\mathrm{p}=0.11)$.

\subsection{Growth and Membrane Integrity}

The effect of farnesol on growth characteristics was subsequently evaluated. In this experiment, the six test strains were inoculated into $0.05 \%$ farnesol in BHI or $\mathrm{BHI}$ alone and incubated for 24 hours at $37^{\circ} \mathrm{C}$. Figure 3 shows that for all strains tested, farnesol decreased the growth yield. While overall the decrease in counts was significant, it appeared that the community isolates appeared more susceptible to farnesol. The average reduction in counts with farnesol for the three resistant strains was $19 \%$, while the reduction in counts averaged $52 \%$ for the community strains.

\subsection{Morphogenesis}

Because of the importance of hyphal growth as a virulence factor for Candida albicans, the effect of farnesol on these organisms was documented microscopically. As shown in Figure 4 the effect on filamentous growth was striking at a concentration of $0.5 \%$ farnesol. This effect was observed with all six of the test strains evaluated and dilutions to $0.125 \%$ farnesol continued to prevent filamentous growth for both drug resistant and community strains. 

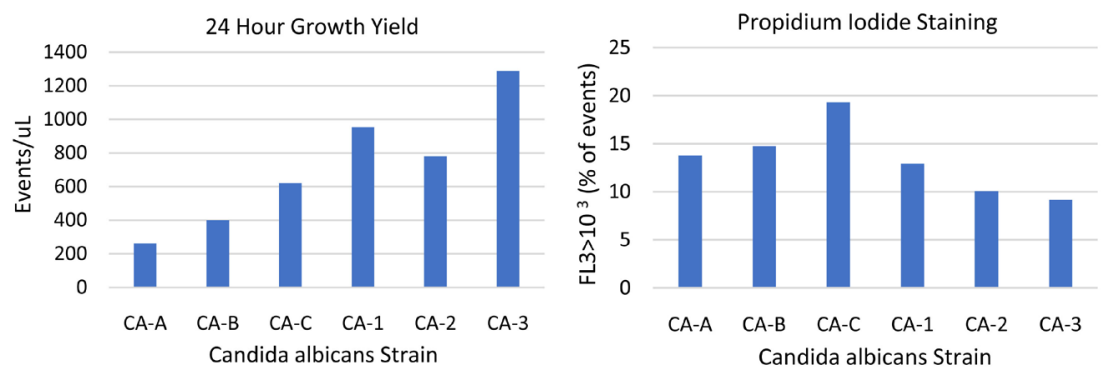

Figure 2. Average values for 3 experiments are shown. Growth after 24 hours is indicated by events/uL in the FSC channel (left). Right panel shows \% of yeast staining with propidum iodide based on fluorescence greater than baseline FL3 signal.

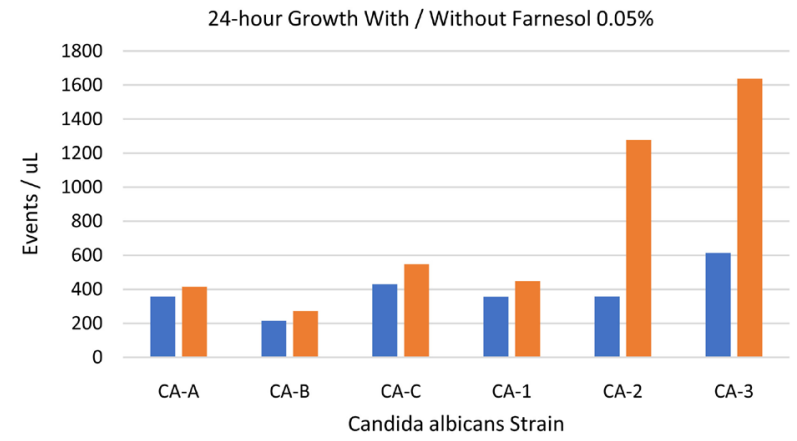

Figure 3. Counts were obtained by flow cytometry and showed that with farnesol (blue bars) consistently produced fewer counts than without farnesol (orange bars). For the entire data set, the difference was significant $(\mathrm{p}=0.05$ paired $\mathrm{T}$ test).

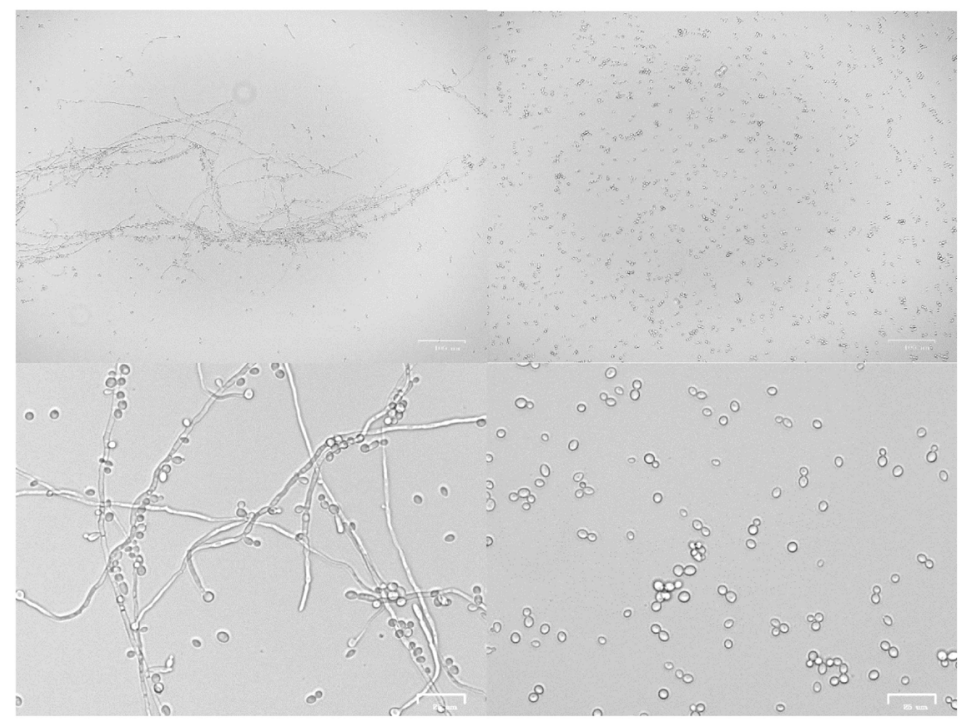

Figure 4. Images of morphology of CA-2 strain of Candida albicans grown for 24 hours in BHI (left panels) or with $0.05 \%$ farnesol in BHI (right panels). Photos were obtained using the BioRad Zoe imager with an initial magnification of approximately $100 \times$ which can be enlarged to approximately $400 \times$. Markers indicate actual scale when printed). Upper panels are wide view to show maximum number of organisms (markers indicate 100 $\mathrm{uM}$ ) and lower panels show detail at higher magnification (markers are $25 \mathrm{uM}$ ). Note that both conditions show yeast with buds and daughter cells indicating that neither had stopped growth. 
These findings aligned with the prior observations in which farnesol added to spider medium inhibited the development of the "spidering" effect seen in the same medium without farnesol.

\subsection{Dose Response}

Hyphal growth limitation persisted at concentrations as low as $0.125 \%$ titration of farnesol and consequently concentrations using serial ten-fold dilutions was employed to determine what concentration was required for diminished growth. Due to the number of samples involved, turbidity measurements were used for this experiment. The highest farnesol content in this experiment was $0.5 \%$. Four serial tenfold dilutions were made to $0.005 \%$ and an intermediate $1: 2$ dilution series $(0.025 \%$ through $0.0025 \%)$ were included for more sensitive endpoint discrimination. As shown in Figure 5, the resistant organisms and community organisms had similar inflection points on the dose-response curve (between 0.05 and $0.025 \%$ ), although the inter-strain variation, not surprisingly, appeared greater with the resistant organisms compared to the community organisms.

\subsection{Stress Response}

While growth was restricted in cultures treated with farnesol, resistance to stress could not necessarily be expected with farnesol treatment. Thus, a heat challenge $\left(50^{\circ} \mathrm{C}\right.$ for 20 minutes) was undertaken to determine if farnesol altered its response to this stressor. The data are presented in Figure 6 shows as expected, all cultures except CA-3 exposed to the heating regimen increased the PI staining, of note, CA-3 in another test (Table 1) did show a 1.9 fold increase in PI staining after heat exposure suggesting this observation was anomalous. To consider the baseline PI staining of each culture, the fold-increase in PI staining before and after heat treatment was employed as an index of the response to heat. Table 2 summarizes these data. The trend shown by this comparison suggests farnesol enhances heat susceptibility but whether the resistant or community organisms are more susceptible to heat is not clear.

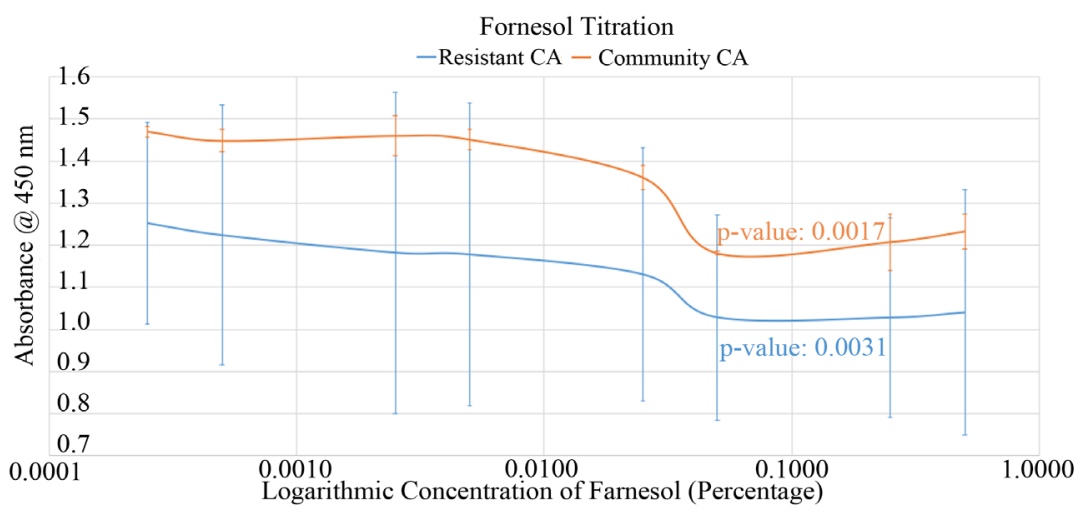

Figure 5. Titration of growth inhibition employed a range of farnesol concentrations and both drug resistant (blue line) and community organisms (orange line) showed a similar inflection point. Signficance was determined by paired $\mathrm{T}$ test comparing the growth in the $0.5 \%$ farnesol and BHI control. 
HEAT STRESS WITHOUT FARNESOL

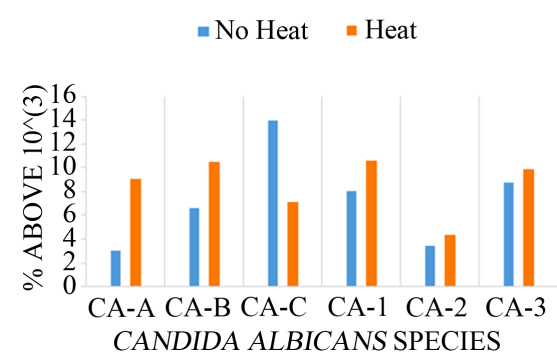

FARNESOL AND HEAT STRESS

-No Heat $\quad$ Heat

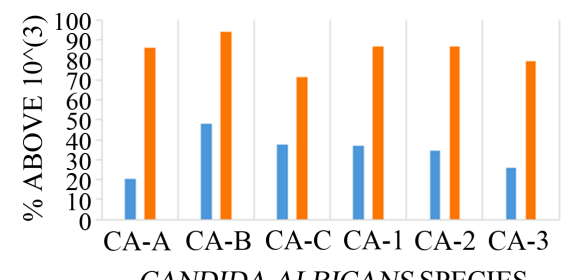

Figure 6. The test organisms were grown without (left panel) or with farnesol (right panel). The baseline PI staining was determined prior to heat treatment (blue bars). The orange bars indicate the PI staining after heat treatment.

Table 2. Heat Stress Index for cultures exposed to farnesol compared to controls not treated with farnesol. The index is fold increase in the PI staining (\% cells with staining intensity above $1 \times 10^{3}$ in the Fl-3 channel) by dividing heated by unheated values. The index for farnesol treated cells compared to controls attained statistical significance ( $\mathrm{p}=$ 0.037 paired $\mathrm{T}$ test).

\begin{tabular}{ccc}
\hline Candidastrain & Control & Farnesol (0.05\%) \\
\hline CA-A & 3.03 & 4.31 \\
CA-B & 1.59 & 1.98 \\
CA-C & 0.51 & 1.9 \\
CA-1 & 1.33 & 2.33 \\
CA-2 & 1.29 & 2.53 \\
CA-3 & 1.13 & 3.08 \\
\hline
\end{tabular}

\subsection{Evaluation of Persisters}

Candida albicans cultures can generate a portion of the population that enters a quiescent state, also known as the persister phenotype. This is associated with organisms sensing the existence of a quorum, when approaching the stationary phase of growth or during environmental stress. Quiescent persister cells are characterized by a condensation of their cytoplasmic material and have been reported to be detectable in a density gradient [10]. In this experiment the indixanol gradients (see methods) were divided into upper and lower fractions with the lower, denser, fraction considered to be enriched with persister and dead cells.

The fractions were separated, reuspended in water and recovered by centrifugation and flow cytometry was used to enumerate the distribution between upper and lower fractions as seen in Figure 7. It may be seen from these data that farnesol increases the proportion of the population found in the lower fraction which is consistent with farnesol pushing organisms toward the persister phenotype.

Because cells were reconstituted quantiatively in water containing propidium iodide, it was possible to simultaneously determine staining that was consistent with dead cells. This is important because persister cells are considered to be still alive though quiescent, and should still have intact membranes that prevent propidum iodide staining. Of note in Figure 8, there appears a tendency for higher PI staining in control versus farnesol exposed cultures which would suggest a greater fraction of farnesol treated cultures harbor persister cells. 


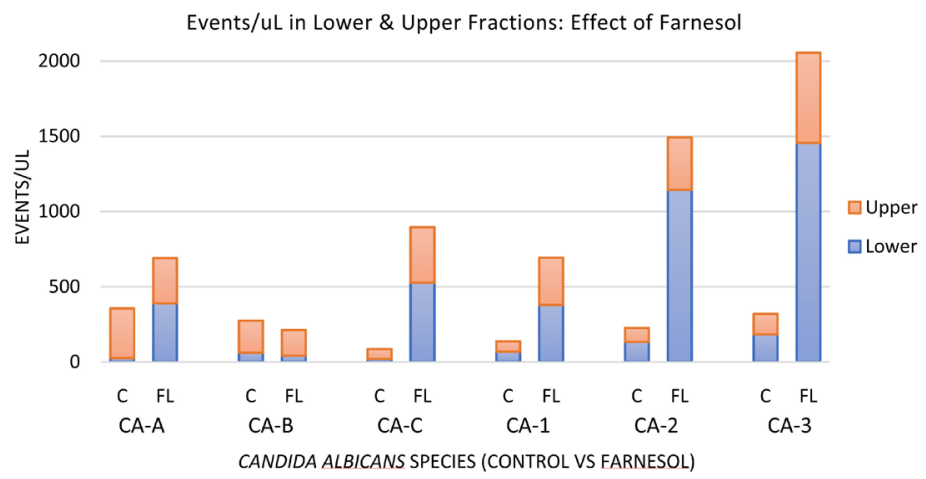

Figure 7. After recovering upper and lower fractions from indixanol separation, each fraction was washed and reconstituted in water containing propidum iodide and cell counts for each fraction were determined in the forward scatter channel of the flow cytometer. Absolute counts from each sample allowed calculation of the percent of the whole population in the lower fraction (see also Table 3).

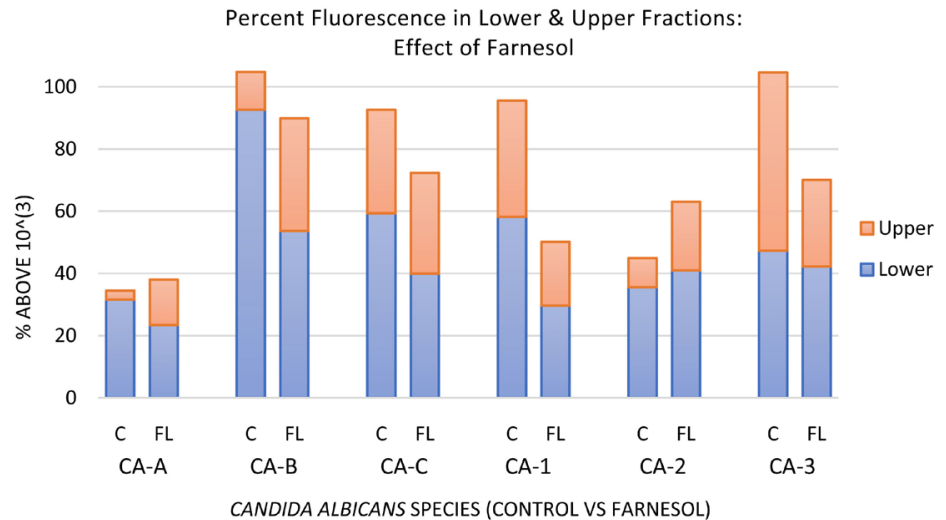

Figure 8. Propidium iodide staining of upper and lower fractions from indixanol separations indicating the percent of events from the upper and lower fractions which stain above the fluorescence threshold.

Table 3. Effect of farnesol on percent of events represented by the lower fraction of indixanol separations.

\begin{tabular}{ccccccc}
\hline & \multicolumn{2}{c}{$\begin{array}{c}\text { \% of culture in } \\
\text { Candida albicans Strain }\end{array}$} & \multicolumn{2}{c}{$\begin{array}{c}\text { \% of cells in lower } \\
\text { fraction not PI stained }\end{array}$} & $\begin{array}{c}\text { Estimated Fraction of } \\
\text { persisters corrected } \\
\text { for dead cells }\end{array}$ \\
\cline { 2 - 7 } & Control & Farnesol & Control & Farnesol & Control & Farnesol \\
\hline CA-A & 3.8 & 51.5 & 61.4 & 76.5 & 0.023 & 0.394 \\
CA-B & 22.6 & 19.7 & 7.4 & 46.3 & 0.017 & 0.091 \\
CA-C & 23.2 & 58.8 & 45.1 & 60 & 0.105 & 0.353 \\
CA-1 & 18.9 & 54.8 & 41.6 & 70.3 & 0.075 & 0.385 \\
CA-2 & 58.8 & 80.6 & 64.4 & 59 & 0.379 & 0.476 \\
CA-3 & 57 & 70.8 & 52.4 & 57.7 & 0.298 & 0.409 \\
\hline
\end{tabular}

${ }^{\star}$ To estimate the fraction of persisters in the lower fraction of the indixanol gradient, the fraction of total cells from the entire sample that were detected in the lower fraction was multiplied by the proportion of cells in the lower fraction that excluded PI. 
This consideration is exploited in Table 3 in which the absolute counts from Figure 7 and Figure 8 are used to predict the percent of the original culture that comprises the persister population. The data in Table 3 supports the likelihood of farnesol promoting persister development.

\section{Discussion}

During its growth, Candida albicans produces the sesquiterpene alcohol farnesol, which among other compounds is an autoinducer of quorum sensing, upregulating virulence genes related to biofilm formation and influencing morphogenesis among many other biological activities [11]. While quorum sensing is a naturally occurring process that helps assure survival, farnesol displays other disparate properties including those directed at human host, modulating immune reactions [12] and enhancing innate immune reactions [13]. Farnesol may also modulate efflux pump activity altering response to antifungal drugs although up or down regulation may relate to concentration [6] [7]. Because of the breadth of biological activities attributed to farnesol, it is reasonable to consider whether exogenously applied to Candida colonized tissues may be beneficial or not.

In these studies, experiments were conducted with a maximum of $0.5 \%$ farnesol. This amount would be equal to $2.25 \times 10^{-2} \mathrm{M} / \mathrm{L}$, however because of its immiscibility, molarity was considered an inappropriate description. For comparison to other literature, however, concentrations reported in various studies ranged from $30 \mathrm{uM}$ to $3 \mathrm{mM}$ [5] [8] [14] [15]. In the present study that range would be covered by the titration (Figure 5) which showed the dose of farnesol that decreased growth yield by $50 \%$ was $0.0035 \%$ which would be comparable to 1 - 2 mM. Other results including suppression of hyphal growth were in consonance with other literature [5].

Two aspects of the current study should be highlighted. First, is by using flow cytometric analysis, it was possible to determine simultaneously the number and relative size of microorganisms evaluated as well as the state of cytoplasmic membrane integrity. The second aspect of this work was the comparison of multi-drug-resistant strains to community strains of Candida albicans. Each of the three drug-resistant was multi-resistant. According to ATCC, the supplier of these strains, CA-A was resistant to anidulafungin, caspofungin, micafungin, voriconazole, itraconazole and fluconazole as well as intermediate resistance to 5-fluorocytosine. CA-B was resistant to anidulafungin and the three afore mentioned triazole antifungals. CA-C had resistance to only two echinocandins, anidulafungin and caspofungin and interestingly, appeared slightly more robust than the test strains with multi-class resistance.

For the entire set of strains, available flow cytometry indicated the drug resistant set appeared less fit than the community organisms in terms of growth yield, relative size of the individual cells, intrinsic viability and viability after heat challenge. For logistical reasons most of the observations were made with subset of the larger culture collection, but some of the important observations suggest 
that it will be worthwhile in the future to expand the number of strains tested in specific experiments, especially in view of the larger standard deviations encountered in the resistant versus community organisms.

These observations will be relevant to the research question engendered by the common use of farnsol as an ingredient in over-the-counter products that may contact tissues colonized by or infected with Candida albicans. Generally, the restriction of growth and hyphal formation would seem beneficial as would the increase in membrane fragility observed both before and after heat challenge. However, this work was expanded to consider the possibility that the quorum sensing activity of farnesol may affect the development of the persister phenotype [16] which could have relevance to Candida survival in or on tissues.

Using a previously reported method [10], farnesol-treated and untreated cultures were separated by density gradient to produce a subpopulation of cells putatively enriched for quiescent persister cells. Because the same fraction was also expected to contain dead cells, the use of propidium iodide staining further characterized the persister rich fraction allowing distinction between active (persister) and inactivated (dead) cells. The ability to use absolute particle (cell) counts allowed an overall estimation of the effect of farnesol on persister development.

In all six of the strains evaluated, farnesol appeared to increase the persister population after 24 hours exposure of the cultures. The overall implication, which will require additional experimental exploration, is that while farnesol may be beneficial as a component of topical products, it may also generate a higher proportion of quiescent persister phenotypes that could prolong colonization or later recrudescence of the organism. Another balancing of activities was reported as farnesol signaling through the RAS pathway both induced higher hydrogen peroxide formation but simultaneously induced systems that detoxify hydrogen peroxide [15].

Researchers have considered whether farnesol might be of value in conditions such as vulvovaginal candidiasis especially because it has also been attributed with biofilm reducing capability [14]. In a study that used a mouse vaginitis model, farnesol alone was not protective and did not benefit infection resolution from fluconazole-sensitive organisms, but some synergy occurred with fluconazole was seen when fluconazole resistant organisms were tested [17].

The potential for benefit in drug resistant cases remains of interest. An older study [18] says farnesol synergized amphotericin B, micafungin and fluconazole in vitro. Another reported fluconazole synergized the effect of fluconazole and 5 -fluorocytosine but antagonized terbinafine and itraconazole. Some molecular mechanisms that may be involved include efflux pump modulation and the related expression of the stress protein CDR-1 [6] [7] and partially explain synergism.

In summary, the potential for the use of farnesol as an inhibitor of Candida albicans alone may have limited value, but its addition to other therapies might have benefits worth exploring. But the potential role of persisters in the colonized tissues and other substances with intrinsic antimicrobial activity in vivo [19] will need to be considered as farnesol is incorporated into consumer products. 
In addition, the focus on farnesol as an additive to consumer products has not delved into the fact that additional quorum sensing mediators exist is Candida and may serve as redundant systems or may simply be overwhelmed in the presence of large concentrations of farnesol. In any case, careful consideration of the biological effects of this and other components of products in contact with colonized tissues should be included in formulation considerations.

\section{Declaration}

The research presented here represents the original work of the authors, all of whom contributed to the planning, research, data collection and reduction and manuscript preparation and review. This manuscript has not been submitted elsewhere and has not previously been published.

\section{Conflicts of Interest}

The authors declare no conflicts of interest regarding the publication of this paper.

\section{References}

[1] Mehmood, A., Liu, G., Wang, X., Meng, G., Wang, C. and Liu, Y. (2019) Fungal Quorum-Sensing Molecules and Inhibitors with Potential Antifungal Activity: A Review. Molecules, 24, 1950. https://doi.org/10.3390/molecules24101950

[2] Polke, M., Leonhardt, I., Kurzai, O. and Jacobsen, I.D. (2018) Farnesol Signalling in Candida albicans-More than Just Communication. Critical Reviews in Microbiology, 44, 230-243. https://doi.org/10.1080/1040841X.2017.1337711

[3] Schnuch, A., Uter, W., Geier, J., Lessmann, H. and Frosch, P.J. (2004) Contact Allergy to Farnesol in 2021 Consecutively Patch Tested Patients. Results of the IVDK. Contact Dermatitis, 50, 117-121. https://doi.org/10.1111/j.0105-1873.2004.0313.x

[4] Nikoomanesh, F., Roudbarmohammadi, S., Khoobi, M., Haghighi, F. and Roudbary, M. (2019) Design and Synthesis of Mucoadhesive Nanogel Containing Farnesol: Investigation of the Effect on HWP1, SAP6 and Rim101 Genes Expression of Candida albicans in Vitro. Artificial Cells, Nanomedicine, and Biotechnology, 47, 64-72. https://doi.org/10.1080/21691401.2018.1543193

[5] Černáková, L., Jordao, L. and Bujdáková, H. (2018) Impact of Farnesol and Corsodyl on Candida albicans Forming Dual Biofilm with Streptococcus Mutans. Oral Diseases, 24, 1126-1131. https://doi.org/10.1111/odi.12873

[6] Černáková, L., Dižová, S., Gášková, D., Jančíková, I. and Bujdáková, H. (2019) Impact of Farnesol as a Modulator of Efflux Pumps in a Fluconazole-Resistant Strain of Candida albicans. Microbial Drug Resistance, 25, 805-812. https://doi.org/10.1089/mdr.2017.0332

[7] Liu, Z., Rossi, J.M. and Myers, L.C. (2018) Candida albicans Zn Cluster Transcription Factors Tacl and Zncl Are Activated by Farnesol to Upregulate a Transcriptional Program Including the Multidrug Efflux Pump CDR1. Antimicrobial Agents and Chemotherapy, 62, e00968-18. https://doi.org/10.1128/AAC.00968-18

[8] Dižová, S., Černáková, L. and Bujdáková, H. (2018) The Impact of Farnesol in Combination with Fluconazole on Candida albicans Biofilm: Regulation of ERG20, ERG9, and ERG11 Genes. Folia Microbiologica, 63, 363-371.

https://doi.org/10.1007/s12223-017-0574-z 
[9] Fernandes Costa, A., Evangelista Araujo, D., Santos Cabral, M., et al. (2019) Development, Characterization, and in Vitro-in Vivo Evaluation of Polymeric Nanoparticles Containing Miconazole and Farnesol for Treatment of Vulvovaginal Candidiasis. Medical Mycology, 57, 52-62. https://doi.org/10.1093/mmy/myx155

[10] Quasem, I., Luby, C.J., Mace, C.R. and Fuchs, S.M. (2017) Density Separation of Quiescent Yeast Using Iodixanol. BioTechniques, 63, 169-173.

https://doi.org/10.2144/000114596

[11] Padder, S.A., Prasad, R. and Shah, A.H. (2018) Quorum Sensing: A Less Known Mode of Communication among Fungi. Microbiological Research, 210, 51-58. https://doi.org/10.1016/j.micres.2018.03.007

[12] Vivas, W., Leonhardt, I., Hünniger, K., Häder, A., Marolda, A. and Kurzai, O. (2019) Multiple Signaling Pathways Involved in Human Dendritic Cell Maturation Are Affected by the Fungal Quorum-Sensing Molecule Farnesol. The Journal of Immunology, 203, 2959-2969. https://doi.org/10.4049/jimmunol.1900431

[13] Zawrotniak, M., Wojtalik, K. and Rapala-Kozik, M. (2019) Farnesol, a Quorum-Sensing Molecule of Candida albicans Triggers the Release of Neutrophil Extracellular Traps. Cells, 8, 1611. https://doi.org/10.3390/cells8121611

[14] Sebaa, S., Boucherit-Otmani, Z. and Courtois, P. (2019) Effects of Tyrosol and Farnesol on Candida albicans Biofilm. Molecular Medicine Reports, 19, 3201-3209. https://doi.org/10.3892/mmr.2019.9981

[15] Deveau, A., Piispanen, A.E., Jackson, A.A. and Hogan, D.A. (2010) Farnesol Induces Hydrogen Peroxide Resistance in Candida albicans Yeast by Inhibiting the Ras-Cyclic AMP Signaling Pathway. Eukaryotic Cell, 9, 569-577. https://doi.org/10.1128/EC.00321-09

[16] Wittman, E., Yar, N. and Larsen, B. (2020) Navigating the Third Frontier of Antimicrobial Therapy to Support Women's Health. Open Journal of Obstetrics and Gynecology, 10, 1011-1035. https://doi.org/10.4236/ojog.2020.1080096

[17] Bozó, A., Domán, M., Majoros, L., Kardos, G., Varga, I. and Kovács, R. (2016) The in Vitro and in Vivo Efficacy of Fluconazole in Combination with Farnesol against Candida albicans Isolates Using a Murine Vulvovaginitis Model. Journal of Microbiology, 54, 753-760. https://doi.org/10.1007/s12275-016-6298-y

[18] Katragkou, A., McCarthy, M., Alexander, E.L., et al. (2015) In Vitro Interactions between Farnesol and Fluconazole, Amphotericin B or Micafungin against Candida albicans Biofilms. Journal of Antimicrobial Chemotherapy, 70, 470-478. https://doi.org/10.1093/jac/dku374

[19] Weber, K., Schulz, B. and Ruhnke, M. (2010) The Quorum-Sensing Molecule E,E-Farnesol-Its Variable Secretion and Its Impact on the Growth and Metabolism of Candida Species. Yeast, 27, 727-739. https://doi.org/10.1002/yea.1769 